\title{
Migración interna y urbanización sin eficiencia en países en desarrollo: evidencia para Ecuador
}

\author{
José Rafael Alvarado-López, Ronny Fabián Correa-Quezada \\ y María del Cisne Tituaña-Castillo
}

\author{
Universidad Nacional de Loja, Ecuador/Universidad Técnica Particular \\ de Loja, Ecuador
}

\section{Resumen}

Ecuador experimenta un acelerado proceso de urbanización en las últimas décadas, causado en parte, por la migración interna. Esto ha provocado una urbanización sin eficiencia ya que la mayor parte de los ingresos provienen de la actividad primaria exportadora y no de las actividades industriales urbanas. En este contexto, el objetivo de esta investigación es estimar los determinantes de la migración interna en Ecuador usando modelos de elección discreta. Los resultados muestran que la fuerza laboral no necesariamente emigra por factores monetarios y que las dotaciones de capital humano reducen la probabilidad de migrar. Una implicación de política pública que se deriva de la presente investigación, es que la migración interna se puede disminuir incrementando la inversión en educación en las regiones periféricas e impulsando la creación de empleo rural.

Palabras clave: Migración interna, capital humano, urbanización, modelos logit, Ecuador.

Abstract

Internal migration and urbanization without efficiency in developing countries: evidence for Ecuador

Ecuador is experiencing an accelerated process of urbanization in recent decades, partly caused by internal migration. This has caused a development without efficiency because most of the revenue comes from primary-export activity rather than urban industrial activities. In this context, the objective of this research is to estimate the determinants of internal migration in Ecuador using discrete choice models. The results show that the labor force will not necessarily migrate by monetary factors and human capital endowments reduce the probability of migrating. A policy implication derived from this research is that internal migration can be reduced by increasing investment in education in peripheral regions and boosting rural employment.

Key words: Internal migration, human capital, urbanisation, logit models, Ecuador. 


\section{INTRODUCCIÓN}

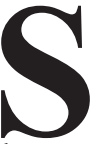

imilar a varios países en vías de desarrollo, el acelerado proceso de urbanización que experimenta la República de Ecuador ha provocado graves problemas socioeconómicos en las grandes ciudades, generando un amplio interés en el ámbito académico y político. Los principales problemas de la expansión urbana en este país son la formación de los cinturones de pobreza, altos niveles de delincuencia, desempleo precario, deficiencia de servicios básicos y educación pública, entre otros.

La literatura teórica señala que la migración es provocada por factores de atracción y de expulsión (Ravenstein, 1885) de las regiones, y por otra, se sostiene que la urbanización aumenta la eficiencia productiva de un país (Henderson, 2001; Gallup, Sachs y Mellinger, 1999). Sin embargo, en varios países en desarrollo y en particular en Ecuador, la urbanización podría resultar ineficiente debido a que las fuerzas de atracción de las ciudades resultan débiles, y en consecuencia la urbanización no podría aumentar la productividad media del país como resultado de la transferencia de mano de obra poco productiva del campo hacia la ciudad, como lo señala Lewis (1974). Al contrario, la migración está transfiriendo mano de obra del sector agrícola y minero, las actividades de mayor productividad, hacia las actividades del área urbana donde existen escases de fuentes de empleo. Además, Henderson (2001) identifica los micro-fundamentas de la relación positiva beneficiosa entre la tasa de urbanización y el crecimiento económico. Uno de los argumentos expuestos por este autor es que la migración interna transfiere fuerza laboral de rendimientos decrecientes desde la actividad agrícola rural hacia la actividad manufacturera de rendimientos crecientes que se localiza principalmente en las grandes ciudades.

Existe una amplia literatura teórica y empírica que señala que las personas migran por las diferencias regionales de salarios, conflictos civiles, pobreza rural, y en particular por la búsqueda de mayores niveles de bienestar que ofrecen las ciudades que son más especializadas (Todaro, 1969; Borjas, 1987; Hoynes, Page y Stevens, 2006). No obstante, en Ecuador, la participación del sector manufacturero en el producto nacional es baja, más bien, una parte significativa de los ingresos provienen de la actividad primaria-exportadora y de los servicios (Alvarado y Atienza, 2014; Alvarado y Toledo, 2017) y no del sector industrial, que en teoría, es el sector que posee las fuerzas de atracción de las ciudades, las que atrae a la fuerza laboral desde el área rural. 
Las investigaciones empíricas al respecto, se han centrado en estimar los determinantes de los flujos migratorios y de la decisión de migrar. En la literatura sobre la migración interna existen varios trabajos que estiman los determinantes de la probabilidad de migrar (Burda, 1993; Jong, 2000; Dahl, 2002; Yankow, 2002; Hunt, 2004; Gries, Kraft y Pieck, 2011;) y los determinantes de los flujos migratorios regionales (Aroca, 2004; Anh, Hoang, Bonfoh y Schelling, 2012; Huy y Khoi, 2011). Una característica transversal del primer grupo de investigaciones es que utilizan logit o probit para estimar el cambio en la probabilidad de migrar de la región a la región cuando cambian las características individuales, familiares y del entorno en las regiones de origen y destino. El segundo grupo de investigaciones utilizan predominantemente econometría de datos de corte transversal y modelos de panel. Las dos metodologías permiten modelar el efecto de los factores de atracción y de expulsión de la región de destino y de origen sobre los flujos migratorios.

Bajo este contexto, el objetivo de esta investigación es examinar los determinantes de las decisiones de migrar utilizando modelos de elección discreta planteados inicialmente por McFadden (1976) y aplicados posteriormente en los trabajos sobre la elección de migrar. En las estimaciones realizadas, consideramos como migrantes a las personas que han migrado en los últimos cinco años al momento de ser encuestados por parte del Instituto Nacional de Estadísticas y Censos. Incluimos los periodos 2000-2004, 2005-2009 y 2010-2014. En esta investigación, un individuo es considerado migrante si cumple una de las siguientes condiciones: que el movimiento migratorio sea del campo a las grandes ciudades o en su efecto, desde las pequeñas ciudades hacia las grandes ciudades. Las principales variables independientes son el ingreso del trabajo y las dotaciones de capital humano de los migrantes. Además, incorporamos un conjunto de covariantes adicionales asociados con las características individuales y familiares de los migrantes. Siguiendo la literatura empírica (Card y DiNardo, 2000; Hanson, 2001; Hoynes, Page y Stevens, 2006;). Asumimos que los trabajadores son racionales y que emigran porque la utilidad que obtienen al migrar es mayor que la utilidad que obtienen al quedarse.

Los resultados encontrados se pueden resumir en dos partes. Primero, encontramos que el salario tiene un efecto estadísticamente poco significativo sobre la decisión de migrar, el resultado no es consistente cuando se incorpora controles adicionales. Segundo, los resultados sugieren que la incorporación de las dotaciones de capital humano y la disponibilidad de un empleo reducen la probabilidad de migrar, y que las fuerzas de atrac- 
ción y repulsión juegan un rol clave para explicar los movimientos migratorios en el territorio. Las recomendaciones de política pública que se derivan de la presente investigación se orientan a que para frenar el acelerado proceso de urbanización y los problemas urbanos antes mencionados, los responsables de la política pueden fomentar una mayor inversión en capital humano en las ciudades periféricas y promover la generación de fuentes de empleo en el área rural.

El resto de esta investigación tiene la siguiente estructura. En la segunda sección se realiza una breve revisión de la literatura teórica y empírica sobre la decisión de migrar y de los flujos migratorios regionales. La tercera sección describe el proceso de urbanización y migración en Ecuador, el volumen y las características de la migración en el país. La cuarta describe las fuentes de datos y la estrategia econométrica. La quinta discute los resultados encontrados y en la sexta constan las conclusiones del trabajo.

\section{ReVisión de LA LITERATURA PREVIA}

La literatura sobre la decisión de migrar y los flujos migratorios dentro de un país es amplia y se ha desarrollado en varias direcciones. Primero, existe una creciente evidencia empírica que muestra que las regiones con mayor nivel de ingreso son más propensas a recibir mayores tasas de inmigración (Huy, 2011; Anh, Hoang, Bonfoh y Schelling, 2012). No obstante, las conclusiones de otras investigaciones señalan que las personas no migran únicamente por el nivel de ingreso, sino por otros factores, tales como el empleo disponible en el lugar de destino (Silva, Guataquí y González, 2007), por las fallas del mercado laboral (Molloy, Smith y Wozniak, 2011), por las redes sociales y familiares (Burda, 1993), entre otras. En este sentido, la literatura muestra la existencia de un conjunto de factores que inciden en las decisiones de migrar. Una parte de estos factores están asociados con las características de los propios migrantes y por factores de expulsión del lugar de origen y factores de atracción en el lugar de destino como lo señala Ravenstein (1985).

Las investigaciones econométricas se fundamentan en la maximización de la función de utilidad del migrante dado su localización y tienen un elemento característico de fondo: las personas migran porque la utilidad que genera localizarse en el lugar de destino es mayor a la utilidad que le genera quedarse en el lugar de residencia. No obstante, las personas no siempre optimizan su utilidad, sino que la migración puede provocar desutilidad o pérdidas sociales (Xiaoling, Li, Zong, Hua, Rong y Abuduhade, 2013; Schündeln, 2014). Aunque la migración urbana-urbana puede jugar 
un rol clave en la asignación del trabajo en el mercado laboral y puede ser relevante su participación en la migración interna (Yap, 1976), el tipo de migración interna que predomina es la migración rural-urbana (Niu y Qi, 2013), al menos en los países en desarrollo. En este sentido, un aspecto clave a considerar en las investigaciones empíricas es la revisión del efecto que produce la migración en las regiones de origen y el impacto que producen los inmigrantes en las ciudades o regiones receptoras. Por una parte, se sugiere que la emigración disminuye la mano de obra de las regiones expulsoras y que la inmigración genera un exceso de oferta de fuerza laboral en las regiones receptoras, pues la migración interna actúa como un mecanismo asignador de la fuerza laboral en el espacio.

Existe evidencia empírica que señala que el acelerado proceso de urbanización que experimentó América Latina (AL) está asociado con la industrialización de la región ocurrida entre las décadas de los 30s y 70s, lo que provocó altos índices de primacía con respecto al resto del mundo (Cerrutti y Bertoncello, 2003). Posteriormente el ritmo de urbanización disminuyó en la mayor parte de los países de la región, lo cual coincide con la pérdida de peso relativo de la actividad industrial en el producto y con el abandono de las políticas de fomento de la industrialización promovido por el modelo de sustitución de importaciones (MSI). Asimismo, Todaro (1980) señala que en AL, los incrementos de la migración interna, campo-ciudad, se intensificó en las décadas de los 1960 y 1970 a pesar de los crecientes niveles de desempleo y subempleo urbano, lo cual puso en duda la validez de los modelos de dos sectores tipo Lewis (1974), donde se argumenta la existencia del efecto positivo que provoca la transferencia de mano de obra poco productiva desde el área rural-agrícola hacia el área urbana-industrial, lo cual aceleraría el desarrollo económico. Este hecho refuerza la premisa de que la migración interna no solo es motivada por factores monetarios, sino también por factores psicológicos. Por ejemplo, se sugiere que la decisión de migrar depende del salario esperado en lugar de las diferencias salariales reales urbano-rural. Estas conclusiones son corroboradas por Mberu (2005), quien señala que no existe ninguna asociación significativa entre la migración interna y la mejora de las condiciones de vida, pues la calidad de vida de los migrantes no es mejor que la de los no migrantes. Por otra parte, se argumenta que los salarios más altos que genera la industria asociada a los rendimientos crecientes con respecto a las actividades primarias asociadas a los rendimientos decrecientes, es un claro factor de movilidad laboral. Además, si la industria está asociada al comercio internacional puede reforzar las decisiones de migrar, como lo 
sugiere $\mathrm{Hu}$ (2002), ya que los migrantes se sienten atraídos por los ingresos que genera el sector industrial-exportador. No obstante, las dos premisas no necesariamente son verdaderas por las características estructurales de los países en desarrollo.

Por otra parte, la migración interna actúa como un mecanismo de asignación de la fuerza laboral entre las actividades en función de su productividad, de eficiencia y de convergencia regional de la renta. Asimismo, los factores de atracción tales como la mayor oferta de empleo en las ciudades, la presencia de las universidades y otras instituciones de capacitación y formación, las instituciones públicas y en general, la presencia de mayores servicios en las grandes ciudades provoca que los migrantes se localicen en las grandes ciudades. Al contrario, las fuerzas de repulsión de migrantes del área rural y pequeñas ciudades son la falta de fuentes de empleo, educación, lugares de diversión y entretenimiento, por lo que migrar parece un mecanismo de relocalización racional; si las fuerzas de repulsión son mayores a las fuerzas de atracción, la migración interna aumenta, y viceversa. Como complementario a esto Rebhun y Goldstein (2009) señalan que las variables socioeconómicas tienen cada vez mayor capacidad explicativa de la migración interna.

Además, un factor relevante a considerar cuando se analiza los determinantes de la migración interna o cuando se diseña políticas públicas para frenarla, es que los más jóvenes son más propensos a migrar, en particular desde las regiones menos desarrolladas hacia regiones con un mayor grado de desarrollo, lo que disminuye la autosuficiencia y la productividad de las regiones expulsoras (Burda, 1993). Por lo tanto, las instituciones educativas y de capacitación pueden jugar un rol central en la retención de los jóvenes en el campo que migran por motivos de estudio y falta de empleo en el área rural. Las recomendaciones de política pública para frenar la migración interna se orientan a promover la eficiente focalización de los subsidios a la vivienda y prohibir la venta de casas (Soto y Torche, 2004). Las políticas orientadas a reducir la migración pueden ser reforzadas con la migración temporal; en este tipo de movilidad, el migrante puede conservar las relaciones familiares, mantener su residencia y comodidades y las actividades tradicionales puede ser una opción para las personas que tienen fuertes lazos con la comunidad, pero pocas fuentes trabajo (Edwards, 2013). Además, este autor señala que la relocalización de las actividades productivas y una importante transformación en los patrones de movilidad de la población pueden reducir los flujos migratorios internos. 
A pesar que los beneficios de la transferencia de mano de obra rural hacia los mercados urbanos es debatible, Jolly, Reeves y Piper (2005) muestran que la migración puede ofrecer nuevas oportunidades para mejorar la vida de la mujer y cambiar las relaciones de opresión de género. Este resultado resulta relevante a partir de que Camlin, Snow y Hosegood (2014) señalan que la migración interna está dominada por las mujeres, impulsada por el aumento de la participación laboral de las mujeres. Se tiene que considerar además que la migración interna puede afectar los empleos locales debido que una mayor afluencia de inmigrantes recientes reduciría la proporción del empleo de los varones nativos significativamente (Berker, 2011), en muchos lugares y en determinados sectores económicos, el incremento de los flujos migratorios reduce las oportunidades laborales de los residentes de dichos territorios (Borjas, 2000).

Resulta lógico pensar que la migración interna genera desequilibrios en los mercados laborales regionales. En el caso de Ecuador, los dos polos de desarrollo, Pichincha y Guayas, donde se localizan Quito y Guayaquil, respectivamente, son las provincias con mayor cantidad de inmigrantes internos. De acuerdo a Loor y Zurita (2017), las provincias con mayor índice de feminidad de los emigrantes son las amazónicas: Morona Santiago, Orellana y Zamora Chinchipe, mientras que Pichincha es la que menor índice presenta. Los procesos de migración interna se relacionan estrictamente con el desempleo y el desarrollo provincial. Las provincias con mayores niveles de inmigrantes son las que tienen menores tasa de desempleo y mayor nivel de desarrollo económico (Vicente y Alvarado, 2017).

\section{Proceso de urbanización y migración interna en Ecuador}

Similar a varios países de América Latina, Ecuador ha experimentado un acelerado proceso de urbanización en las últimas décadas. En este proceso, la localización de la actividad económica juega un rol clave porque direcciona los procesos migratorios al interior de un país. La Figura 1 muestra la evolución de la población rural y urbana desde 1960 hasta el 2016. Por una parte, se puede evidenciar que la población urbana como porcentaje del total aumenta y la población rural como porcentaje del total disminuye de forma acelerada. Para el caso de Ecuador, en 1983 la población urbana ya superó a la rural, cuando en América Latina este fenómeno ocurrió en 1993 aproximadamente, mientras que a nivel global, la población urbana superó a la población rural apenas en el 2007 (Banco Mundial, 2017). Estos hechos destacan el acelerado proceso de urbanización que siguió Ecuador, y dicho proceso es reforzado por la migración del campo a la ciudad. 


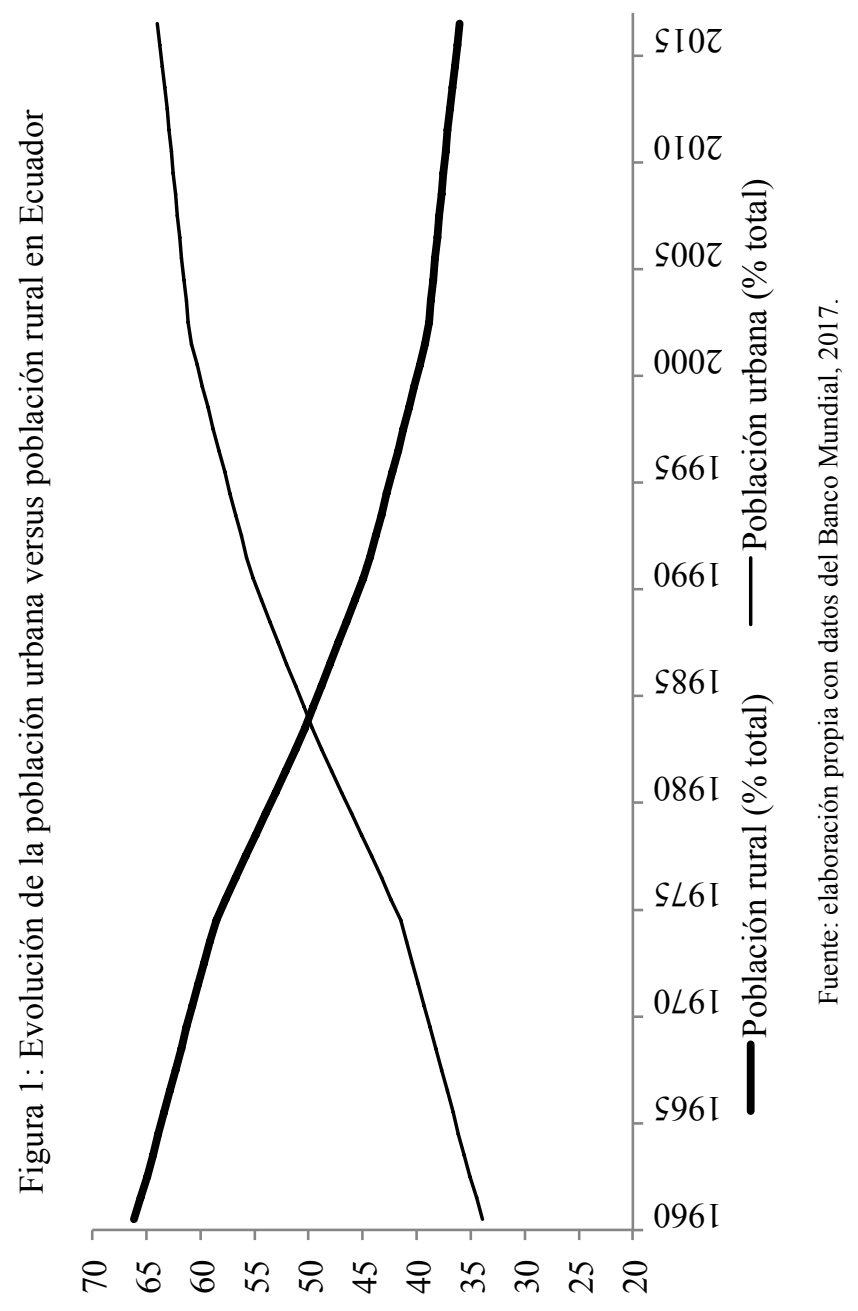


En Ecuador las aglomeraciones urbanas de la industria y la infraestructura generaron efectos acumulativos en determinados núcleos de crecimiento económico y demográfico (Correa, 2016); las provincias con mayor dinamismo atraen más inmigrantes y las provincias con menor actividad económica expulsan más emigrantes. Además, existen otros factores que pueden contribuir a explicar la migración interna, tales como la colonización de la Amazonía y la localización de las actividades de extracción de petróleo y la actividad turística de las Islas Galápagos (Tabla 1).

Tabla 1: Distribución territorial de la migración interna en Ecuador en el 2010

\begin{tabular}{lrrr}
\hline \multicolumn{1}{c}{ Provincia } & Inmigrantes & Emigrantes & Migración neta \\
\hline Azuay & 29594 & 21167 & 8427 \\
Bolívar & 6855 & 14191 & -7336 \\
Cañar & 8285 & 7775 & 510 \\
Carchi & 7658 & 13914 & -6256 \\
Cotopaxi & 15661 & 21937 & -6276 \\
Chimborazo & 17608 & 25721 & -8113 \\
El Oro & 27934 & 29090 & -1156 \\
Esmeraldas & 26939 & 38455 & -11516 \\
Guayas & 89561 & 81890 & 7671 \\
Imbabura & 17510 & 18831 & -1321 \\
Loja & 18523 & 27137 & -8614 \\
Los Ríos & 32131 & 38947 & -6816 \\
Manabí & 39422 & 65718 & -26296 \\
Morona S. & 8559 & 8263 & 296 \\
Napo & 6213 & 6212 & 1 \\
Pastaza & 9098 & 5921 & 3177 \\
Pichincha & 138269 & 92657 & 45612 \\
Tungurahua & 18501 & 19494 & -993 \\
Zamora Ch. & 6498 & 6225 & 273 \\
Galápagos & 3557 & 2429 & 1128 \\
Sucumbíos & 14135 & 14664 & -529 \\
Orellana & 14396 & 6835 & 7561 \\
Santo D. & 33390 & 29317 & 4073 \\
Santa Elena & 14769 & 8276 & 6493 \\
\hline
\end{tabular}

Fuente: elaboración propia con datos del INEC, 2017. 
La columna migración neta muestra la diferencia entre los inmigrantes y los migrantes por cada provincia obtenido del Censo de Población y Vivienda de 2010, el cual es el último Censo realizado en este país. De las 24 provincias, 12 tienen una migración neta positiva (provincias receptoras) y la otra mitad una tasa de migración neta negativa (provincias expulsoras). Este proceso genera que las provincias receptoras cuenten con mayor fuerza laboral y en las provincias expulsoras la fuerza laboral disminuya.

\section{Datos Y Metodología}

\section{Datos}

Los datos utilizados en esta investigación provienen de la Encuesta Nacional de Empleo, Desempleo y Subempleo (ENEMDU) de 2004, 2009 y 2014 publicada por el Instituto Nacional de Estadísticas y Censos (INEC) de Ecuador. Esta Base de datos proporciona información estadística del mercado laboral urbano y rural con cobertura nacional (excluye Galápagos para 2004 y 2009) y sigue las recomendaciones de la Organización Internacional del Trabajo (OIT). Consideramos como migrantes a las personas que han migrado en los últimos cinco años; en este sentido, la encuesta de 2004 abarca a los que migraron entre 2000-2004, la encuesta de 2009 abarca a los migrantes de 2005-2009 y la encuesta del 2014 abarca a los migrantes de 2010-2014. Asimismo, la información permite incorporar las principales características de los encuestados. La Tabla 2 resume la descripción de las variables con sus estadísticos descriptivos para los tres periodos analizados. Con lo cual examinamos empíricamente los determinantes de la migración interna en Ecuador. El modelo estimado se expone en la siguiente sección.

En todas las regresiones la variable dependiente es dicótoma, $1 \mathrm{si} \mathrm{mi-}$ gra, 0 en otro caso. La principal variable explicativa es el salario y las dotaciones de capital humano de los migrantes (escolaridad, experiencia, edad). La lógica subyacente de la inclusión del salario como la principal variable que explica la migración interna es por dos razones. La primera es que las dos provincias con mayor nivel de ingresos y entre las que cuentan con menor desempleo son las que mayor inmigración presentan. Paralelamente, las provincias con mayores niveles de emigrantes son aquellas que tienen bajos niveles de ingresos y mayores tasas de desempleo. La segunda es que la evidencia empírica parece sugerir que los factores monetarios siguen jugando un rol clave en la determinación de la decisión de migrar. 
Migración interna y urbanización sin eficiencia en paises en desarrollo: evidencia para Ecuador / R. ALVARADO et al.

Tabla 2: Estadísticos descriptivos de las variables utilizadas

\begin{tabular}{|c|c|c|c|c|c|}
\hline Estadísticos descriptivos 2000-2004 & Obs. & Media & DS & Min. & Max. \\
\hline Migración* (1 migrante, 0 No migrante) & 16211 & 0.0576769 & 0.2331386 & 0 & 1 \\
\hline Sexo (1 hombre, 0 mujer) & 16211 & 0.6741719 & 0.4686979 & 0 & 1 \\
\hline Salario & 16211 & 184.8719 & 244.3286 & 0 & 8650 \\
\hline Edad & 16211 & 34.23749 & 12.5284 & 15 & 65 \\
\hline Edad al cuadrado & 16211 & 1329.157 & 945.964 & 225 & 4225 \\
\hline Escolaridad & 16211 & 9.657455 & 5.285967 & 0 & 21 \\
\hline Experiencia & 16211 & 9.185553 & 10.35471 & 0 & 55 \\
\hline Experiencia al cuadrado & 16211 & 191.5877 & 368.7386 & 0 & 3025 \\
\hline Trabajo (1 Si, 0 No) & 16211 & 0.9830979 & 0.1289087 & 0 & 1 \\
\hline Seguro Social (1 IESS ISFA, 0 Otro caso) & 16211 & 0.2947381 & 0.455939 & 0 & 1 \\
\hline Jefe de hogar (1 Si, 0 Otro caso) & 16211 & 0.4353834 & 0.4958224 & 0 & 1 \\
\hline Etnia (1 Indígena, 2 Blanco, 3 Mestizo, 4 Negro) & 16211 & 2.834187 & 0.5587228 & 1 & 4 \\
\hline Sector (1 Primario, 2 Manufactura, 3 Servicios) & 16211 & 2.27469 & 0.9014282 & 1 & 3 \\
\hline \multicolumn{6}{|l|}{ Estadísticos descriptivos 2005-2009 } \\
\hline Migración* (1 migrante, 0 No migrante) & 12488 & 0.0542921 & 0.2266023 & 0 & 1 \\
\hline Sexo (1 hombre, 0 mujer) & 12488 & 0.5570948 & 0.4967494 & 0 & 1 \\
\hline Salario & 12488 & 257.853 & 267.8639 & 0 & 5000 \\
\hline Edad & 12488 & 35.73286 & 12.76028 & 15 & 65 \\
\hline Edad al cuadrado & 12488 & 1439.649 & 988.706 & 225 & 4225 \\
\hline Escolaridad & 12488 & 10.76642 & 5.217582 & 0 & 21 \\
\hline Experiencia & 11761 & 8.816682 & 10.24496 & 0 & 60 \\
\hline Experiencia al cuadrado & 11761 & 182.6842 & 357.0113 & 0 & 3600 \\
\hline Título (1 Si, 0 No) & 12488 & 0.170804 & 0.3763527 & 0 & 1 \\
\hline Trabajo (1 Si, 0 No) & 12488 & 0.9240871 & 0.2648693 & 0 & 1 \\
\hline Seguro Social (1 IESS ISFA, 0 Otro caso) & 12488 & .3646701 & 0.4813568 & 0 & 1 \\
\hline Jefe de hogar (1 Si, 0 Otro caso) & 12488 & 0.4188821 & 0.4933958 & 0 & 1 \\
\hline Etnia (1 Indígena, 2 Blanco, 3 Mestizo, 4 Negro) & 12488 & 2.8746 & 0.5068303 & 1 & 4 \\
\hline Sector (1 Primario, 2 Manufactura, 3 Servicios) & 12488 & 2.410714 & 0.8520039 & 1 & 3 \\
\hline \multicolumn{6}{|l|}{ Estadísticos descriptivos 2010-2014 } \\
\hline Migración* (1 migrante, 0 No migrante) & 26478 & 0.06 & 0.252 & 0 & 1 \\
\hline Salario & 26478 & 429.35 & 370.94 & 0 & 5000 \\
\hline Sexo (1 hombre, 0 mujer $)$ & 26478 & 0.65 & 0.47 & 0 & 1 \\
\hline Edad & 26478 & 35.82 & 12.11 & 15 & 65,0 \\
\hline Edad al cuadrado & 26478 & 1430.13 & 943.03 & 225 & 4225 \\
\hline Escolaridad & 26478 & 11.31 & 4.96 & 0 & 21 \\
\hline Experiencia & 25358 & 8.05 & 9.63 & 0 & 55 \\
\hline Experiencia al cuadrado & 25358 & 157.85 & 328.9 & 0 & 3025 \\
\hline Título (1 Si, 0 No) & 26478 & 0.18 & 0.38 & 0 & 1 \\
\hline Trabajo (1 Si, 0 No) & 26478 & 0.94 & 0.22 & 0 & 1 \\
\hline Seguro Social (1 IESS ISFA, 0 Otro caso) & 26478 & 0.54 & 0.49 & 0 & 1 \\
\hline Jefe de hogar (1 Si, 0 Otro caso) & 26478 & 0.474 & 0.49 & 0 & 1 \\
\hline Etnia (1 Indígena, 2 Negro, 3 Mestizo, 4 Blanco) & 26478 & 2.82 & 0.56 & 1 & 4 \\
\hline Sector (1 Primario, 2 Manufactura, 3 Servicios) & 26478 & 2.41 & 0.87 & 1 & 3 \\
\hline
\end{tabular}

Fuente: elaboración propia con datos del INEC. 
En este contexto, se plantea la hipótesis de que el salario tiene un efecto positivo en la decisión de migrar. En resumen, el acelerado proceso de urbanización de Ecuador es reforzado por la migración interna, la cual es causada en parte por las diferencias espaciales de ingresos: las ciudades con mayores niveles de salarios atraen más inmigrantes y las ciudades con menores salarios expulsan mayores niveles de emigrantes.

Además, se incluye diversas covariantes asociadas con las características de carácter general de los encuestados tales como: sexo, salario, edad, título, seguridad social, jefe de hogar, etnia y sector económico. La inclusión de éstas variables permite visualizar el perfil social, demográfico y económico de la población. Asimismo, contribuye a conseguir estimadores consistentes y robustos, y en particular a capturar la estructura del mercado laboral ecuatoriano y las diferencias en la cobertura a la seguridad social, la etnicidad y la estructura productiva del país.

A partir de los datos utilizados, nuestras principales hipótesis sobre el resto de las variables se plantean a continuación:

- Se espera que la variable sexo del migrante influya en la decisión de migrar, que los hombres migren más que las mujeres. Esta hipótesis está fundamentada en la evidencia empírica que plantea Loor y Zurita (2017) para el caso ecuatoriano.

- Se plantea la hipótesis de que la migración aumenta con la edad pero a una tasa decreciente. Esta hipótesis está basada en el análisis de los datos para el caso de estudio.

- Resulta lógico esperar que las personas con mayor capital humano y experiencia cuenten con mayor facilidad para encontrar un empleo, por lo que pueden moverse en el territorio. Al respecto, Faggian y McCann (2009) señalan que el capital humano más calificado es más móvil en el espacio. En este sentido, se espera un efecto positivo para la variable escolaridad, experiencia, y título profesional. En esta dirección, es posible que la concentración de las instituciones educativas refuerce la migración interna hacia las grandes ciudades Quito y Guayaquil (Cueva y Alvarado, 2017).

- La variable si tiene trabajo una persona debería motivar a quedarse y a migrar si está desempleado.

- La variable jefe de hogar debería incidir de forma negativa en la elección de migrar.

- La seguridad social es una variable institucional que captura la estabilidad laboral y formalidad de un contrato laboral por lo que se espera que tenga un efecto negativo en la elección de migrar. 
- Finalmente, las variables etnia y sector, se espera que los trabajadores indígenas, negros, y los trabajadores del sector primario tengan mayores probabilidades de migrar. Esta hipótesis se basa en que la población indígena y negra se localiza en las provincias más pobres del país. Similarmente, se espera que los trabajadores del área rural que se especializan principalmente en el sector primario sean más propensos a migrar.

\section{Metodología}

Los modelos de elección discreta desarrollados inicialmente por McFadden (1976) se han utilizado ampliamente para modelar la decisión de migrar. A partir de estos modelos, se puede formalizar la utilidad de migrar en función de las características individuales de la fuerza laboral y de las condiciones de la región de origen y de destino (Aroca, 2004). En este sentido, utilizamos este marco instrumental con el fin de estimar los determinantes de la migración interna. Primero, estimamos el efecto del salario y de las dotaciones de capital humano en la probabilidad de migrar y luego realizamos un conjunto de regresiones controlado por las variables antes mencionadas mediante modelos de logit. La ecuación (1) formaliza el modelo estimado.

$$
\mathrm{P}_{\mathrm{i}}=\alpha_{0}+\alpha_{1} \mathrm{~W}_{\mathrm{i}}+\alpha_{2} \mathrm{CH}_{\mathrm{i}}+\alpha_{3} \mathrm{Z}_{\mathrm{i}}+\gamma_{\mathrm{i}}
$$

Donde $\mathrm{P}_{\mathrm{i}}$ mide la probabilidad de que el individuo $i$ migre y que cumpla las condiciones antes expuestas, $\mathrm{W}_{\mathrm{i}}$ es el salario del individuo, $\mathrm{CH}_{\mathrm{i}}$ son las dotaciones de capital humano de los migrantes, $Z_{\mathrm{i}}$ representa un conjunto de controles antes descrito. Finalmente, $\mathrm{g}_{\mathrm{i}}$ es el término de error estocástico del modelo, el cual asumimos que sigue una distribución binomial. Los resultados de la estimación de la ecuación (1) se discuten a continuación.

\section{Discusión de Resultados}

La Tabla 3 muestra los resultados del modelo (1) estimado para el periodo 2000-2004, la misma que muestra que el efecto del salario sobre la probabilidad de migrar no tiene un efecto significativo en todas las regresiones, más bien se vuelve no significativo cuando se incorporan los controles: empleo, acceso a la seguridad social, la etnicidad y el sector productivo, en tres regresiones el salario motiva la migración en forma positiva. 
Tabla 3: Resultados modelo logit de migración 2000-2004

\begin{tabular}{|c|c|c|c|c|c|}
\hline & [1] & [2] & [3] & [4] & [5] \\
\hline $\begin{array}{l}\text { Sexo } \\
\end{array}$ & 0.142 & 0.144 & $0.148^{*}$ & -0.155 & $-0.176^{*}$ \\
\hline & $(1.89)$ & $(1.91)$ & & & \\
\hline Salario & $0.000263^{*}$ & $0.000266^{*}$ & $0.000237^{*}$ & 0.000184 & 0.000194 \\
\hline Edad & $\begin{array}{r}(2.31) \\
0.0980^{* * *}\end{array}$ & $\begin{array}{r}(2.34) \\
0.0988^{* * * *}\end{array}$ & $\begin{array}{r}(2.01) \\
0.0955^{* * * *}\end{array}$ & $\begin{array}{r}(1.50) \\
0.0362\end{array}$ & $\begin{array}{r}(1.59) \\
0.0360\end{array}$ \\
\hline & $(5.13)$ & $(5.15)$ & $(4.95)$ & $(1.80)$ & $(1.78)$ \\
\hline Edad2 & $\begin{array}{r}-0.00149^{* * * *} \\
(-5.40)\end{array}$ & $\begin{array}{r}-0.00150^{* * * *} \\
(-5.43)\end{array}$ & $\begin{array}{r}-0.00148^{* * *} \\
(-5.35)\end{array}$ & $\begin{array}{r}-0.000910^{* *} \\
(-3.22)\end{array}$ & $\begin{array}{r}-0.000911^{* *} \\
(-3.22)\end{array}$ \\
\hline Escolaridad & $\begin{array}{r}-0.0367^{* * * *} \\
(-4.99)\end{array}$ & $\begin{array}{r}-0.0367^{* * *} \\
(-4.99)\end{array}$ & $\begin{array}{r}-0.0463^{* * * *} \\
(-5.78)\end{array}$ & $\begin{array}{r}-0.0385^{* * *} \\
(-4.75)\end{array}$ & $\begin{array}{r}-0.0362^{* * *} \\
(-4.18)\end{array}$ \\
\hline Experiencia & $\begin{array}{l}-0.146^{* * *} \\
(-12.89)\end{array}$ & $\begin{array}{c}-0.146^{* * *} \\
(-12.89)\end{array}$ & $\begin{array}{r}-0.151^{* * *} \\
(-13.19)\end{array}$ & $\begin{array}{r}-0.152^{* * *} \\
(-13.27)\end{array}$ & $\begin{array}{r}-0.156^{* * *} \\
(-13.53)\end{array}$ \\
\hline Experiencia2 & $\begin{array}{r}0.00288^{* * * *} \\
(8.72)\end{array}$ & $\begin{array}{r}0.00289^{* * *} \\
(8.72)\end{array}$ & $\begin{array}{r}0.00301^{* * *} \\
(9.08)\end{array}$ & $\begin{array}{r}0.00302^{* * *} \\
(9.14)\end{array}$ & $\begin{array}{r}0.00307^{* * *} \\
(9.29)\end{array}$ \\
\hline Trabaja (1=Si) & & $\begin{array}{r}-0.279 \\
(-1.17)\end{array}$ & $\begin{array}{l}-0.290 \\
(-1.22)\end{array}$ & $\begin{array}{l}-0.294 \\
(-1.23)\end{array}$ & $\begin{array}{r}-0.280 \\
(-1.17)\end{array}$ \\
\hline S. social $(1=\mathrm{Si})$ & & & $\begin{array}{r}0.281^{* * *} \\
(3.13)\end{array}$ & $\begin{array}{l}0.225^{*} \\
(2.50)\end{array}$ & $\begin{array}{r}0.262^{* *} \\
(2.90)\end{array}$ \\
\hline Jefe de hogar $(1=\mathrm{Si})$ & & & & $\begin{array}{r}0.885^{* * 4} \\
(9.84)\end{array}$ & $\begin{array}{r}0.885^{* * *} \\
(9.81)\end{array}$ \\
\hline Blanco & & & & & $\begin{array}{l}0.438^{*} \\
(2.40)\end{array}$ \\
\hline Mestizo & & & & & $\begin{array}{r}0.173 \\
(1.19)\end{array}$ \\
\hline Negro & & & & & $\begin{array}{r}0.0553 \\
(0.22)\end{array}$ \\
\hline Manufactura & & & & & $\begin{array}{r}-0.598^{* * * *} \\
(-4.47)\end{array}$ \\
\hline Servicios & & & & & $\begin{array}{l}-0.166 \\
(-1.92)\end{array}$ \\
\hline Constant & $\begin{array}{c}-3.374^{* * *} \\
(-11.01)\end{array}$ & $\begin{array}{r}-3.111^{* * *} \\
(-8.21)\end{array}$ & $\begin{array}{r}-2.979^{* * *} \\
(-7.81)\end{array}$ & $\begin{array}{r}-1.988^{* * *} \\
(-5.05)\end{array}$ & $\begin{array}{r}-2.015^{* * * *} \\
(-4.90)\end{array}$ \\
\hline Observations & 16208 & 16208 & 16208 & 16208 & 16208 \\
\hline
\end{tabular}

Los resultados encontrados en las tres primeras regresiones son similares a las conclusiones obtenidas por Anh, Hoang, Bonfoh y Schelling, 2012 y Huy (2011), quienes señalan que el nivel de ingreso de las regiones receptoras juega un rol clave en la determinación de la decisión de migrar. Los resultados de las dos últimas regresiones, tras la incorporación sugieren que el nivel salarial no puede explicar por si solo la decisión de migrar. Estos resultados concuerdan con las ideas que proponen Silva, Guataquí y González (2007), quienes señalan que la decisión de migrar depende en gran medida del empleo disponible en las regiones receptoras. 
Efectivamente, las provincias con menores tasas de desempleo son las que reciben más inmigrantes en el periodo analizado.

Por otra parte, en todos los modelos de regresión, el efecto de la edad, la escolaridad y la experiencia, con sus respectivos valores cuadráticos son significativos al 0.1 por ciento. En la práctica, este resultado implica que las personas migran más con la edad y con la sus habilidades observables medido por la experiencia laboral, pero migra menos ante el incremento del capital humano. Este resultado es relevante desde el punto de vista de la política de retención de los migrantes en el área rural y en las pequeñas ciudades, ya que si aumenta la inversión en capital humano en estos lugares, las personas van a migrar en menor medida. En el caso de Ecuador, las personas con mayores niveles de capital humano son menos propensas a migrar. Este resultado es contrario al encontrado por Faggian y McCann (2009) en países desarrollados.

El resultado de la variable edad es similar al salario. Es estadísticamente significativo en las tres primeras regresiones y pierde capacidad explicativa en las dos últimas regresiones. El efecto de la edad al cuadrado es estadísticamente significativo, influye de manera negativa en la decisión de migrar a medida que aumenta la edad del trabajador. Este resultado sugiere que las personas migran en mayor medida hasta una cierta edad y luego decrece. La variable seguridad social tiene un efecto positivo en la decisión de migrar. De igual forma, los jefes de hogar son más propensos a migrar. Finalmente, un resultado de interés es que la etnia de comparación que son los indígenas es menos propensa a migrar con respecto a sus pares de las otras etnias. Y se verifica la hipótesis de que los trabajadores del sector primario son más propensos a migrar que los trabajadores del sector manufacturero y de los servicios.

En la Tabla 4 se observa que a diferencia del período anterior que el efecto del salario sobre la probabilidad de migrar es significativo en todas las regresiones. A medida que el salario aumenta la probabilidad de migrar también aumenta. En todas las regresiones, el capital humano (escolaridad, experiencia) disminuye la probabilidad de migrar y cuando aumenta la edad la probabilidad de migrar aumenta y este resultado es estadísticamente significativo. Este resultado refuerza la conclusión obtenida con las estimaciones presentados en la Tabla 3. Además, la variable título se incluyó en este periodo por la disponibilidad de la información y se puede evidenciar que la misma no es estadísticamente significativa en todas las regresiones. Finalmente, se encuentra que los jefes de hogar son más propensos a migrar, lo mismo ocurre con las personas que trabajan en el sector manufacturero, los jefes de hogar y los mestizos. 
Tabla 4: Resultados modelo logit de migración 2005-2009

\begin{tabular}{|c|c|c|c|c|c|}
\hline & {$[1]$} & [2] & [3] & [4] & [5] \\
\hline Sexo & $\begin{array}{r}0.328^{* * * *} \\
(3.69)\end{array}$ & $\begin{array}{c}0.328^{* * * *} \\
(3.70)\end{array}$ & $\begin{array}{r}0.328^{* * 4 *} \\
(3.70)\end{array}$ & $\begin{array}{r}-0.0670 \\
(-0.70)\end{array}$ & $\begin{array}{r}-0.0675 \\
(-0.69)\end{array}$ \\
\hline Salario & $\begin{array}{r}0.000811^{* * *} \\
(5.75)\end{array}$ & $\begin{array}{r}0.000814^{* * *} \\
(5.77)\end{array}$ & $\begin{array}{r}0.000773^{* * * *} \\
(5.39)\end{array}$ & $\begin{array}{r}0.000658^{* * * *} \\
(4.50)\end{array}$ & $\begin{array}{r}0.000645^{* * *} \\
(4.39)\end{array}$ \\
\hline Edad & $\begin{array}{r}0.0941^{* * * *} \\
(3.69)\end{array}$ & $\begin{array}{r}0.0946^{* * * *} \\
(3.71)\end{array}$ & $\begin{array}{r}0.0891^{\text {**** }} \\
(3.47)\end{array}$ & $\begin{array}{r}0.0148 \\
(0.56)\end{array}$ & $\begin{array}{r}0.0179 \\
(0.67)\end{array}$ \\
\hline Edad2 & $\begin{array}{r}-0.00171^{* * *} \\
(-4.68)\end{array}$ & $\begin{array}{r}-0.00172^{* * *} \\
(-4.70)\end{array}$ & $\begin{array}{r}-0.00166^{* * *} \\
(-4.52)\end{array}$ & $\begin{array}{r}-0.000936^{*} \\
(-2.53)\end{array}$ & $\begin{array}{r}-0.000981^{* *} \\
(-2.63)\end{array}$ \\
\hline Escolaridad & $\begin{array}{r}-0.0288^{* *} \\
(-2.60)\end{array}$ & $\begin{array}{r}-0.0287^{* *} \\
(-2.59)\end{array}$ & $\begin{array}{r}-0.0338^{* *} \\
(-2.95)\end{array}$ & $\begin{array}{r}-0.0242^{*} \\
(-2.07)\end{array}$ & $\begin{array}{r}-0.0175 \\
(-1.44)\end{array}$ \\
\hline Experiencia & $\begin{array}{r}-0.130^{* * * *} \\
(-9.08)\end{array}$ & $\begin{array}{r}-0.130^{* * *} \\
(-9.09)\end{array}$ & $\begin{array}{r}-0.132^{* * * *} \\
(-9.18)\end{array}$ & $\begin{array}{r}-0.133^{* * *} \\
(-9.19)\end{array}$ & $\begin{array}{r}-0.135^{* * * *} \\
(-9.32)\end{array}$ \\
\hline Experiencia2 & $\begin{array}{r}0.00291^{* * * *} \\
(6.80)\end{array}$ & $\begin{array}{r}0.00291^{* * * *} \\
(6.80)\end{array}$ & $\begin{array}{r}0.00296^{* * *} \\
(6.93)\end{array}$ & $\begin{array}{r}0.00295^{* * * *} \\
(6.92)\end{array}$ & $\begin{array}{r}0.00299^{* * *} \\
(7.02)\end{array}$ \\
\hline Titulo (1=Si) & $\begin{array}{r}-0.0679 \\
(-0.42)\end{array}$ & $\begin{array}{r}-0.0668 \\
(-0.41)\end{array}$ & $\begin{array}{r}-0.0857 \\
(-0.53)\end{array}$ & $\begin{array}{r}-0.0203 \\
(-0.12)\end{array}$ & $\begin{array}{r}-0.0828 \\
(-0.50)\end{array}$ \\
\hline Trabaja $(1=\mathrm{Si})$ & & $\begin{array}{l}-0.259 \\
(-0.94)\end{array}$ & $\begin{array}{l}-0.269 \\
(-0.98)\end{array}$ & $\begin{array}{l}-0.288 \\
(-1.04)\end{array}$ & $\begin{array}{l}-0.279 \\
(-1.01)\end{array}$ \\
\hline S. social $81=\mathrm{Si}$ ) & & & $\begin{array}{l}0.175 \\
(1.75)\end{array}$ & $\begin{array}{r}0.157 \\
(1.56)\end{array}$ & $\begin{array}{l}0.206^{*} \\
(2.02)\end{array}$ \\
\hline Jefe $(1=\mathrm{Si})$ & & & & $\begin{array}{r}1.197^{* * * *} \\
(11.35)\end{array}$ & $\begin{array}{r}1.194^{* * * *} \\
(11.25)\end{array}$ \\
\hline Blanco & & & & & $\begin{array}{l}0.437^{*} \\
(2.03)\end{array}$ \\
\hline Mestizo & & & & & $\begin{array}{l}-0.370^{*} \\
(-2.15)\end{array}$ \\
\hline Negro & & & & & $\begin{array}{r}0.184 \\
(0.66)\end{array}$ \\
\hline Manufactura & & & & & $\begin{array}{r}-0.517^{* * *} \\
(-3.08)\end{array}$ \\
\hline Servicios & & & & & $\begin{array}{l}-0.167 \\
(-1.54)\end{array}$ \\
\hline Constant & $\begin{array}{r}-3.489^{* * *} \\
(-7.92) \\
\end{array}$ & $\begin{array}{r}-3.247^{* * *} \\
(-6.38) \\
\end{array}$ & $\begin{array}{r}-3.120^{* * *} \\
(-6.07) \\
\end{array}$ & $\begin{array}{r}-1.941^{* * *} \\
(-3.70) \\
\end{array}$ & $\begin{array}{r}-1.630^{* *} \\
(-3.03) \\
\end{array}$ \\
\hline $\begin{array}{l}\text { Observations } \\
\text { Adjusted } R^{2}\end{array}$ & 11761 & 11761 & 11761 & 11761 & 11761 \\
\hline
\end{tabular}

En esta misma dirección, la Tabla 5 reporta los resultados del modelo logit para el periodo 2010-2014. Similar a los resultados obtenidos en la Tabla 4, el salario aumenta la probabilidad de que una persona migre, el resultado es consistente a pesar de la inclusión de controles. El resultado se mantiene para la escolaridad y la experiencia como medidas de capital humano, es decir, a medida que aumenta el capital humano, la probabilidad de migrar disminuye. Al contrario, a medida que aumenta la edad, las 
personas migran en mayor medida. Si el encuestado es jefe de hogar tiene mayores probabilidad de migrar y en este periodo, el poseer un título profesional si es estadísticamente significativo, si las personas son profesionales, tienen mayor probabilidad de migrar. Otras investigaciones recientes destacan que la migración interna ya no necesariamente se concentra en las grandes ciudades (Quito y Guayaquil), sino que las ciudades intermedias son cada vez más importantes (Rayuela y Ordoñez, 2016).

Tabla 5: Resultados modelo logit de migración 2010-2014

\begin{tabular}{|c|c|c|c|c|c|}
\hline & {$[1]$} & [2] & [3] & [4] & [5] \\
\hline \multirow[t]{2}{*}{ Sexo } & $0.268^{\text {**** }}$ & $0.269^{2 * * k^{*}}$ & $0.269^{2 * 4 * 3 *}$ & 0.0226 & 0.0253 \\
\hline & $(4.79)$ & (4.79) & $(4.80)$ & $(0.38)$ & $(0.42)$ \\
\hline \multirow[t]{2}{*}{ Salario } & 0.000000280 & 0.000000283 & 0.000000285 & 0.000000376 & 0.000000342 \\
\hline & $(0.54)$ & $(0.54)$ & $(0.55)$ & $(0.72)$ & $(0.65)$ \\
\hline \multirow[t]{2}{*}{ Edad } & $0.0700^{* * * *}$ & $0.0699^{* * *}$ & $0.0710^{* * *}$ & 0.0180 & 0.0174 \\
\hline & $(4.52)$ & $(4.51)$ & $(4.53)$ & (1.11) & (1.07) \\
\hline \multirow[t]{2}{*}{ Edad2 } & $-0.00114^{* * *}$ & $-0.00113^{* * *}$ & $-0.00115^{* * *}$ & $-0.000625^{* *}$ & $-0.000628^{* *}$ \\
\hline & $(-5.33)$ & $(-5.33)$ & $(-5.35)$ & $(-2.88)$ & $(-2.89)$ \\
\hline \multirow[t]{2}{*}{ Escolaridad } & $-0.0165^{*}$ & $-0.0166^{*}$ & $-0.0157^{*}$ & -0.0102 & -0.0120 \\
\hline & $(-2.36)$ & $(-2.36)$ & $(-2.16)$ & $(-1.39)$ & $(-1.60)$ \\
\hline \multirow[t]{2}{*}{ Experiencia } & $-0.109^{* * *}$ & $-0.109^{* * *}$ & $-0.109^{* * *}$ & $-0.111^{* * *}$ & $-0.111^{* * *}$ \\
\hline & $(-12.21)$ & $(-12.20)$ & $(-12.20)$ & $(-12.44)$ & $(-12.33)$ \\
\hline \multirow[t]{2}{*}{ Experiencia2 } & $0.00209^{* * *}$ & $0.00209^{* * *}$ & $0.00209^{* * *}$ & $0.00212^{* * *}$ & $0.00210^{* * *}$ \\
\hline & $(7.45)$ & (7.44) & (7.41) & $(7.55)$ & (7.48) \\
\hline \multirow[t]{2}{*}{ Titulo (1=Si) } & $0.182^{*}$ & $0.183^{*}$ & $0.187^{*}$ & $0.245^{* *}$ & $0.240^{* *}$ \\
\hline & $(2.02)$ & $(2.03)$ & $(2.07)$ & $(2.68)$ & $(2.62)$ \\
\hline \multirow[t]{2}{*}{ Trabaja $(1=\mathrm{Si})$} & & -0.264 & -0.262 & -0.216 & -0.221 \\
\hline & & $(-1.27)$ & $(-1.25)$ & $(-1.03)$ & $(-1.05)$ \\
\hline \multirow[t]{2}{*}{ S. $\operatorname{social}(1=\mathrm{Si})$} & & & -0.0256 & -0.0526 & -0.0515 \\
\hline & & & $(-0.45)$ & $(-0.92)$ & $(-0.90)$ \\
\hline \multirow[t]{2}{*}{ Jefe $(1=\mathrm{Si})$} & & & & $0.777^{* * * *}$ & $0.783^{* * *}$ \\
\hline & & & & $(12.62)$ & (12.71) \\
\hline \multirow[t]{2}{*}{ Negro y afro } & & & & & 0.132 \\
\hline & & & & & $(0.79)$ \\
\hline \multirow[t]{2}{*}{ Mestizo } & & & & & 0.194 \\
\hline & & & & & (1.95) \\
\hline \multirow[t]{2}{*}{ Blanco } & & & & & $1.035^{* * *}$ \\
\hline & & & & & $(5.51)$ \\
\hline \multirow[t]{2}{*}{ Manufactura } & & & & & -0.0591 \\
\hline & & & & & $(-0.52)$ \\
\hline \multirow[t]{2}{*}{ Servicios } & & & & & 0.0138 \\
\hline & & & & & $(0.22)$ \\
\hline \multirow[t]{2}{*}{ Constant } & $-3.125^{* * *}$ & $-2.862^{* * *}$ & $-2.882^{* * *}$ & $-2.072^{* * *}$ & $-2.229^{* * * *}$ \\
\hline & $(-11.12)$ & $(-8.19)$ & $(-8.18)$ & $(-5.78)$ & $(-6.07)$ \\
\hline $\begin{array}{l}\text { Observations } \\
\text { Adjusted } R^{2}\end{array}$ & 25424 & 25424 & 25424 & 25424 & 25424 \\
\hline
\end{tabular}


Nuestra investigación ofrece tres resultados de interés. Primero, se encuentra que el salario tiene un efecto positivo en la decisión de migrar pero pierde capacidad explicativa cuando se incorporan las variables que capturan las características estructurales del país: la diversidad étnica y la especialización productiva. Estos resultados son coherentes parcialmente con las conclusiones obtenidas por Rebhun y Goldstein (2009), pero la migración interna puede generar pérdidas en las provincias expulsoras como lo señala Burda (1993) y otras investigaciones recientes (Schündeln, 2014; Xiaoling et al., 2013). Segundo, se encuentra que las variables relacionadas con el capital humano pueden ser mecanismo clave para reducir la migración interna, en particular en las regiones que la emigración expulsa la mano de obra productiva y genera disminución de capacidad productiva en ellas. Efectivamente, la migración interna se ha reducido en los últimos años como resultado del aumento de la inversión en capital humano y otros gastos sociales realizados por el Estado en la última década. Tercero, existe una escasa movilidad espacial de los indígenas con respecto al resto de etnias. Y como cabía esperar, los trabajadores que se especializan en el sector primario son más propensos a migrar, lo cual refuerza la idea de que en los países en desarrollo, en particular en los países de América Latina, la migración campo ciudad ocurre porque en las ciudades existen mayores fuentes de empleo en la manufactura y los servicios.

\section{Conclusiones}

En esta investigación estimamos los determinantes de la migración interna en Ecuador usando modelos de elección discreta para el periodo 20002004, 2005-2009 y 2010-2014 a nivel de microdatos. Lo resultados indican que el salario influye de forma positiva en la probabilidad de migrar, aunque el resultado no es consistente para el primer periodo analizado, pero si es consistente en los otros dos periodos. El segundo resultado de interés es que el capital humano (escolaridad y experiencia) reduce la probabilidad de migrar, en todas las regresiones, el coeficiente asociado a estas variables son estadísticamente significativos. A partir de la medida de migración incluida en esta investigación, en términos de política pública, un mecanismo para retener a las personas en las pequeñas ciudades y en el área rural, la política debería enfocarse en aumentar la inversión en capital humano en las regiones periféricas. Las políticas orientadas a la generación de empleo rural también podrían contribuir a la retención de la fuerza laboral en el campo. Futuras investigaciones para el caso ecuatoriano podrían centrase en una mayor formalización del espacio en el estudio de la migración 
interna y en la modelación de los flujos migratorios ya que permitirían comprender de forma más integral los procesos que llevan a las personas a moverse en el territorio.

\section{REFERENCIAS BIBLIOGRÁFICAS}

Alvarado, R. y Atienza, M., 2014, The role of market access and human capital in regional wage disparities: Empirical evidence for Ecuador, Universidad Católica del Norte, Chile, Department of Economics.

Alvarado, R. y Toledo, E., 2017, Environmental degradation and economic growth: evidence for a developing country, in Environment, Development and Sustainability, 19(4), 1205-1218.

Anh, L. T., Hoang, V. L., Bonfoh, B. y Schelling, E., 2012, "An analysis of interprovincial migration in Vietnam from 1989 to 2009", in Global Health Action, 5, 1-12.

Aroca, P., 2004, "Migración interregional en Chile. Modelos y resultados 19872002", en Notas de población, (78), 97-154.

Berker, A., 2011, "Labor-market consequences of internal migration in Turkey", in Economic Development and Cultural Change, 60(1), 197-239

Borjas, G. J., 1987, "Economic theory and international migration", in International migration Review, 457-485.

Borjas, G. J., 2000, "Ethnic enclaves and assimilation", in Swedish Economic Policy Review, 7(2), 89-122.

Burda, M. C., 1993, The determinants of East-West German migration: Some first results, in European Economic Review, 37(2-3), 452-461.

Camlin, C. S., Snow, R. C., \& Hosegood, V., 2014, Gendered patterns of migration in rural South Africa, in Population, space and place, 20(6), 528-551.

Card, D. y DiNardo, J. E., 2000, Do immigrant inflows lead to native outflows?, National Bureau of Economic Research.

Cerrutti, M., \& Bertoncello, R., 2003, Urbanization and internal migration patterns in Latin America, Centro de Estudios de Población. Argentina, 1-24.

Correa, R., 2016, "Las políticas de desarrollo regional en Ecuador", en Yachana, Revista Científica, 5(2), 150-165.

Cueva, K. y Alvarado, R., 2017, "Concentración espacial de capital humano calificado y desigualdad regional de ingresos en Ecuador", en Revista Paradigma Económico 9(1), 5-26.

Dahl, G. B., 2002, "Mobility and the return to education: Testing a Roy model with multiple markets", in Econometrica, 70(6), 2367-2420. 
Edwards, W., 2013, “Temporary Internal Migration: Inferences from Survey Data", in Journal of Northern Studies, 7(2), 75-93.

Faggian, A. y McCann, P., 2009, "Human capital and regional development", in R. Capello y P. Nijkamp (eds.), Handbook of regional growth and development theories (pp. 133-151). Edward Elgar Publishing Limited.

Gallup, J. L., Sachs, J. D. y Mellinger, A. D., 1999, "Geography and economic development", in International Regional Science Review, 22(2), 179-232.

Gries, T., Kraft, M. y Pieck, C., 2011, "Interregional migration, self-selection and the returns to education in Brazil", in The Annals of Regional Science, 46(3), 707732 .

Hanson, G. H., 2001, "US-Mexico integration and regional economies: evidence from border-city pairs", in Journal of Urban Economics, 50(2), 259-287.

Henderson, V., 2001, "Urbanization in Developing Countries", in World Bank Research Observer, vol. 17, 1, pp. 89-112.

Hoynes, H. W., Page, M. E. y Stevens, A. H., 2006, "Poverty in America: Trends and explanations", in The Journal of Economic Perspectives, 47-68.

$\mathrm{Hu}, \mathrm{D} ., 2002$, Trade, rural-urban migration, and regional income disparity in developing countries: a spatial general equilibrium model inspired by the case of China, in Regional Science and Urban Economics, 32(3), 311-338.

Hunt, J., 2004, “Are migrants more skilled than non-migrants? Repeat, return, and same-employer migrants", in Canadian Journal of Economics/Revue canadienne d'économique, 37(4), 830-849

Huy, H. T., 2011, "Analysis of labour migration flows in the Mekong Delta of Vietnam", in Environmental change and agricultural sustainability in the Mekong Delta (pp. 115-140). Springer Netherlands.

Huy, H. T. y Khoi, L. N. D., 2011, "Analysis of Labour Migration Flows in the Mekong Delta of Vietnam", in Environmental Change and Agricultural Sustainability in the Mekong Delta, 115-140.

Jolly, S., Reeves, H., \& Piper, N., 2005, Gender and migration: Overview report. Bridge Development - Gender. Working Paper.

Jong, G.F.D., 2000, "Expectations, gender, and norms in migration decision-making", in Popul Stud 54(3): 307-319

Lewis, H. G., 1974, "Comments on selectivity biases in wage comparisons", in The Journal of Political Economy 1145-1155.

Loor, K. y Zurita, G., 2017, Estadísticas y Distribución Espacial de la Migración en el Ecuador según Censo 2010, Bachelor's Thesis, ESPOL.

Mberu, B. U., 2005, "Internal migration and household living conditions in Ethiopia", in African population and Health Center.

McFadden, D., 1976, "Quantal Choice Analysis: A Survey", in Annals of Economics and Social Measurement, 363-390. 
Molloy, R., Smith, C. L., y Wozniak, A., 2011, I"nternal migration in the United States", in The Journal of Economic Perspectives, 25(3), 173-196.

Niu, J., y Qi, Y., 2013, "Population Migration and Health Stratification in Urban China", in XXVII IUSSP International Population Conference, Busan, August. Working Paper.

Ravenstein, E. G., 1885, "The laws of migration", in Journal of the Statistical Society of London, 167-235.

Rebhun, U., y Goldstein, S., 2009, “Dynamics of Internal Migration Determinants for American Jews, 1985-1990 and 1995-2000", in Population Research and Policy Review, 28(2), 143-167.

Schündeln, M., 2014, "Are immigrants more mobile than natives? Evidence from Germany”, in Journal of Regional Science, 54(1), 70-95.

Silva, C., Guataquí Roa, J. C., y González Román, P., 2007, “The effect of internal migration on the Colombian labor market", in Global Journal of Business Research, 1(1), 70-82.

Soto, R., y Torche, A., 2004, "Spatial inequality, migration and economic growth in Chile", in Cuadernos de Economía, 41(124), 401-424.

Todaro, M. P., 1969, “A model of labor migration and urban unemployment in less developed countries", in The American Economic Review, 138-148.

Todaro, M., 1980, "Internal migration in developing countries: a survey", in Population and economic change in developing countries (pp. 361-402). University of Chicago Press.

Vicente, I. y Alvarado, R., 2017, Determinantes del desempleo juvenil regional en países en vías de desarrollo: un enfoque logit multinivel para Ecuador. Documento de Trabajo. Universidad Nacional de Loja. Loja, Ecuador.

Xiaoling, E; Li, P. S.; Zong, L. Hua W.; Rong, W. y Abuduhade, 2013, “Integration of minority migrant workers in Lanzhou, China", in Canadian Ethnic Studies, 45(3), 117-131.

Yankow, J. J., 2002, The Geographic Mobility of Displaced Workers: Do Local Labour Market Conditions Matter. Furman University Discussion Paper.

Yap, L. Y., 1976, "Rural-urban migration and urban underemployment in Brazil", in Journal of Development Economics, 3(3), 227-243. 


\section{RESUMEN CURRICULAR DE LOS AUTORES}

\section{José Rafael Alvarado López}

Es Docente e Investigador de la Escuela de Economía de la Universidad Nacional de Loja (UNL), en Ecuador. Investiga y publica en temas relacionados con la desigualdad regional, sector externo y crecimiento económico.

Dirección electrónica: rafaalvaradolopez@gmail.com

\section{Ronny Fabián Correa Quezada}

Es Docente e Investigador del Departamento de Economía de la Universidad Técnica Particular de Loja (UTPL), en Ecuador. Ha escrito capítulos de libros y varias publicaciones indexadas y artículos divulgativos.

Dirección electrónica: rfcorrea@utpl.edu.ec

\section{María del Cisne Tituaña Castillo}

Es Docente e Investigadora del Departamento de Economía de la Universidad Técnica Particular de Loja (UTPL), en Ecuador. Los campos de interés son la migración interna e internacional, economía regional, desarrollo económico y social, economía del bienestar y desigualdad.

Dirección electrónica: mdtituania@utpl.edu.ec

Artículo recibido el 4 de septiembre de 2015 y aprobado el 16 de junio de 2017. 
Migración interna y urbanización sin eficiencia en países en desarrollo: evidencia para Ecuador / R. ALVARADO et al.

Anexo 1: Efectos marginales periodo 2000-2004

\begin{tabular}{lccccc}
\hline & {$[1]$} & {$[2]$} & {$[3]$} & {$[4]$} & {$[5]$} \\
\hline Sexo & 0.0065343 & 0.0065915 & 0.0067185 & -0.0070891 & -0.0078691 \\
& $(2.01)$ & $(2.03)$ & $(2.07)$ & $(-1.90)$ & $(-2.07)$ \\
Salario & 0.0000122 & 0.0000123 & 0.000011 & 0.00000817 & 0.00000826 \\
& $(2.33)$ & $(2.35)$ & $(2.05)$ & $(1.53)$ & $(1.56)$ \\
Edad & 0.0043221 & 0.0043423 & 0.0041814 & 0.0013787 & 0.0014134 \\
& $(5.12)$ & $(5.14)$ & $(4.94)$ & $(1.60)$ & $(1.64)$ \\
Edad2 & -0.0000663 & -0.0000666 & -0.0000655 & -0.0000372 & -0.0000372 \\
& $(-5.46)$ & $(-5.48)$ & $(-5.39)$ & $(-3.09)$ & $(-3.11)$ \\
Escolaridad & -0.0018251 & -0.0018255 & -0.0022156 & -0.0017804 & -0.001616 \\
& $(-5.53)$ & $(-5.53)$ & $(-6.21)$ & $(-5.12)$ & $(-4.40)$ \\
Experiencia & -0.0065578 & -0.006556 & -0.0067343 & -0.0064953 & -0.006555 \\
& $(-13.69)$ & $(-13.69)$ & $(-14.00)$ & $(-14.02)$ & $(-14.13)$ \\
Experiencia2 & 0.0001263 & 0.0001263 & 0.0001312 & 0.0001261 & 0.0001264 \\
& $(8.51)$ & $(8.51)$ & $(8.85)$ & $(8.91)$ & $(8.97)$ \\
Trabaja (1=Si) & & -0.0105571 & -0.0112174 & -0.0111629 & -0.0109867 \\
& & $(-0.82)$ & $(-0.86)$ & $(-0.89)$ & $(-0.87)$ \\
S. social (1=Si) & & & 0.0122742 & 0.0089606 & 0.0092231 \\
& & & $(2.77)$ & $(2.15)$ & $(2.21)$ \\
Jefe de hogar $(1=S i)$ & & & & 0.0420177 & 0.0420425 \\
& & & & $(9.45)$ & $(9.45)$ \\
Etnia & & & & & -0.0007344 \\
& & & & & $(-0.29)$ \\
Rama & & & & & -0.0025355 \\
& & & & & $(-1.36)$ \\
\hline Observaciones & 16780 & & & & 1678780 \\
\hline Fuente: elaboración de los autores. & & & &
\end{tabular}


Anexo 2: Efectos marginales periodo 2005-2009

\begin{tabular}{lccccc}
\hline & {$[1]$} & {$[2]$} & {$[3]$} & {$[4]$} & {$[5]$} \\
\hline Sexo & 0.0064621 & 0.0064763 & 0.0065498 & -0.0067156 & -0.007224 \\
& $(2.22)$ & $(2.23)$ & $(2.26)$ & $(-2.16)$ & $(-2.29)$ \\
Salario & 0.0000315 & 0.0000315 & 0.0000301 & 0.0000222 & 0.000022 \\
& $(7.24)$ & $(7.24)$ & $(6.82)$ & $(5.47)$ & $(5.43)$ \\
Edad & 0.0027751 & 0.0027792 & 0.0025434 & -0.0005428 & -0.0004145 \\
& $(3.55)$ & $(3.56)$ & $(3.22)$ & $(-0.72)$ & $(-0.55)$ \\
Edad2 & -0.0000495 & -0.0000496 & -0.000047 & -0.0000159 & -0.000017 \\
& $(-4.51)$ & $(-4.51)$ & $(-4.25)$ & $(-1.55)$ & $(-1.67)$ \\
Escolaridad & -0.0006149 & -0.0006132 & -0.0008229 & -0.0003711 & -0.0002005 \\
& $(-1.71)$ & $(-1.71)$ & $(-2.22)$ & $(-1.09)$ & $(-0.58)$ \\
Experiencia & -0.0044718 & -0.0044719 & -0.0045277 & -0.0040631 & -0.0041077 \\
& $(-9.89)$ & $(-9.89)$ & $(-10.03)$ & $(-9.86)$ & $(-10.02)$ \\
Experiencia2 & 0.0000919 & 0.0000919 & 0.0000936 & 0.0000831 & 0.0000836 \\
& $(6.51)$ & $(6.51)$ & $(6.67)$ & $(6.55)$ & $(6.64)$ \\
Titulo (1=Si) & -0.0085588 & -0.008557 & -0.0092083 & -0.0055491 & -0.0061175 \\
& $(-1.79)$ & $(-1.79)$ & $(-1.96)$ & $(-1.23)$ & $(-1.38)$ \\
Trabaja $(1=S i)$ & & -0.0027147 & -0.0031689 & -0.0026847 & -0.0023219 \\
& & $(-0.25)$ & $(-0.29)$ & $(-0.27)$ & $(-0.24)$ \\
S. social $81=S i)$ & & & 0.0073083 & 0.0058678 & 0.006399 \\
& & & $(2.12)$ & $(1.89)$ & $(2.05)$ \\
Jefe (1=Si) & & & & 0.0520605 & 0.0517862 \\
& & & & $(12.55)$ & $(12.50)$ \\
Etnia & & & & & -0.0059672 \\
& & & & & $(-2.81)$ \\
Rama & & & & & -0.0020409 \\
& & & & & $1.33)$ \\
\hline Observaciones & 17120 & 17120 & 17120 & 17120 & 17120 \\
\hline & & & & &
\end{tabular}

Fuente: elaboración de los autores. 
Migración interna y urbanización sin eficiencia en países en desarrollo: evidencia para Ecuador / R. ALVARADO et al.

Anexo 3: Efectos marginales periodo 2010-2014

\begin{tabular}{lccccc}
\hline & {$[1]$} & {$[2]$} & {$[3]$} & {$[4]$} & {$[5]$} \\
\hline Sexo & 0.0112913 & 0.0113014 & 0.0112149 & -0.001317 & -0.0012253 \\
& $(3.84)$ & $(3.85)$ & $(3.82)$ & $(0.41)$ & $(-0.38)$ \\
Salario & 0.0000252 & 0.0000252 & 0.0000266 & 0.0000226 & 0.0000222 \\
& $(6.45)$ & $(6.46)$ & $(6.75)$ & $(5.83)$ & $(5.73)$ \\
Edad & 0.0033704 & 0.0033602 & 0.0035631 & 0.0007485 & 0.0006849 \\
& $(3.96)$ & $(3.95)$ & $(4.16)$ & $(0.87)$ & $(0.80)$ \\
Edad2 & -0.0000586 & -0.0000584 & -0.0000607 & -0.000032 & -0.0000314 \\
& $-(-5.04)$ & $(-5.03)$ & $(-5.20)$ & $(-2.78)$ & $(-2.73)$ \\
Escolaridad & -0.001476 & -0.0014776 & -0.0013165 & -0.0009434 & -0.0010248 \\
& $(-3.75)$ & $(-3.75)$ & $(-3.26)$ & $(-2.40)$ & $(-2.56)$ \\
Experiencia & -0.0061604 & -0.006155 & -0.0061467 & -0.0060146 & -0.0060051 \\
& $(-12.96)$ & $(-12.95)$ & $(-12.93)$ & $(-13.13)$ & $(-13.10)$ \\
Experiencia2 & 0.00012 & 0.0001199 & 0.0001189 & 0.0001155 & 0.0001152 \\
& $(7.82)$ & $(7.81)$ & $(7.73)$ & $(7.82)$ & $(7.81)$ \\
Titulo (1=Si) & 0.0021369 & 0.002215 & 0.002482 & 0.0063524 & 0.0065948 \\
& $(0.40)$ & $(0.42)$ & $(0.47)$ & $(1.18)$ & $(1.23)$ \\
Trabaja (1=Si) & & -0.0168911 & -0.016226 & -0.0127322 & -0.0133293 \\
& & $(-1.17)$ & $(-1.13)$ & $(-0.95)$ & $(-0.99)$ \\
S. social(1=Si) & & & -0.0055987 & -0.0063073 & -0.0066064 \\
& & & $(-1.72)$ & $(-2.00)$ & $(-2.10)$ \\
Jefe (1=Si) & & & & 0.0409128 & 0.0412812 \\
& & & & $(11.90)$ & $(12.02)$ \\
Etnia & & & & & 0.0071306 \\
& & & & & $(2.87)$ \\
Rama & & & & & 0.0001395 \\
& & & & & $(0.08)$ \\
\hline Observaciones & 25358 & 25358 & 25358 & 25358 & 25358 \\
\hline Fon & & & & &
\end{tabular}

Fuente: elaboración de los autores. 